\title{
S-nitrosoglutathione reductases are low-copy number, cysteine-rich proteins in plants that control multiple developmental and defense responses in Arabidopsis
}

\author{
Shengbao $\mathrm{Xu}^{1,2+\neq}$, Damian Guerra ${ }^{1 \neq}$, Ung Lee $^{3 \dagger}$ and Elizabeth Vierling ${ }^{1 *}$ \\ ' Department of Biochemistry and Molecular Biology, University of Massachusetts-Amherst, Amherst, MA, USA \\ 2 School of Life Sciences, Lanzhou University, Gansu, China \\ ${ }^{3}$ Department of Biochemistry and Molecular Biophysics, University of Arizona, Tucson, AZ, USA
}

\section{Edited by:}

John Hancock, University of the

West of England, Bristol, UK

\section{Reviewed by:}

Joerg Durner, Helmholtz Center Munich, Germany

Joshua Hare, University of Miami Miller School of Medicine, USA

\section{*Correspondence:}

Elizabeth Vierling, Life Science Laboratories, University of

Massachusetts-Amherst,

240 Thatcher Road, Amherst, MA

01003, USA

e-mail: vierling@biochem.umass.edu

${ }^{\dagger}$ Present address:

Shengbao Xu, School of Life

Sciences, Lanzhou University, Lanzhou, Gansu, China;

Ung Lee, Global Education \&

Training Services LLC, Reston, USA

${ }^{\ddagger}$ These authors have contributed

equally to this work.
S-nitrosoglutathione reductase (GSNOR) is believed to modulate effects of reactive oxygen and nitrogen species through catabolism of S-nitrosoglutathione (GSNO). We combined bioinformatics of plant GSNOR genes, localization of GSNOR in Arabidopsis thaliana, and microarray analysis of a GSNOR null mutant to gain insights into the function and regulation of this critical enzyme in nitric oxide (NO) homeostasis. GSNOR-encoding genes are known to have high homology across diverse eukaryotic taxa, but contributions of specific conserved residues have not been assessed. With bioinformatics and structural modeling, we show that plant GSNORs likely localize to the cytosol, contain conserved, solvent-accessible cysteines, and tend to be encoded by a single gene. Arabidopsis thaliana homozygous for GSNOR loss-of-function alleles exhibited defects in stem and trichome branching, and complementation with Green fluorescent protein (GFP) -tagged GSNOR under control of the native promoter quantitatively rescued these phenotypes. GSNOR-GFP showed fluorescence throughout Arabidopsis seedlings, consistent with ubiquitous expression of the protein, but with especially high fluorescence in the root tip, apical meristem, and flowers. At the cellular level we observed cytosolic and nuclear fluorescence, with exclusion from the nucleolus. Microarray analysis identified 99 up- and 170 down-regulated genes ( $\geq 2$-fold; $p \leq 0.01$ ) in a GSNOR null mutant compared to wild type. Six members of the plant specific, ROXY glutaredoxins and three BHLH transcription factors involved in iron homeostasis were strongly upregulated, supporting a role for GSNOR in redox and iron metabolism. One third of downregulated genes are linked to pathogen resistance, providing further basis for the reported pathogen sensitivity of GSNOR null mutants. Together, these findings indicate GSNOR regulates multiple developmental and metabolic programs in plants and offer insight into putative routes of post-translational GSNOR regulation.

Keywords: S-nitrosoglutathione (GSNO), S-nitrosoglutathione reductase (GSNOR), nitrosative stress, trichomes, nitric oxide homeostasis, formaldehyde metabolism, glutaredoxin, pathogen defense

\section{INTRODUCTION}

In plants, biological processes ranging from leaf stomatal closure to auxin perception in roots and pathogen infection involve nitric oxide (NO) (Neill et al., 2002; Floryszak-Wieczorek et al., 2007; Lozano-Juste and Leon, 2011; Terrile et al., 2012). While NO itself is ostensibly active, it is also thought to be transmitted to distal targets via low molecular weight S-nitrosothiols (SNOs), of which the glutathione (GSH) adduct S-nitrosoglutathione (GSNO) is the most abundant (Broniowska et al., 2013; Corpas et al., 2013). GSNO can profoundly affect protein activity through glutathionylation and nitrosation of cysteines (Romeo et al., 2002; Giustarini et al., 2005; Zaffagnini et al., 2013), implying that cells require mechanisms to spatiotemporally control GSNO levels. Catabolism of GSNO by S-nitrosoglutathione reductase (GSNOR) is common to eukaryotes and many bacteria and is believed to be responsible for this regulation of
GSNO levels (Liu et al., 2001; Staab et al., 2008). GSNOR exhibits NAD/H-dependent oxidoreductase activity toward a broad spectrum of aliphatic compounds, but its preferred substrates are GSNO and S-hydroxymethylglutathione (HMGSH), an intermediate in formaldehyde metabolism (Jensen et al., 1998; Achkor et al., 2003; Kubienová et al., 2013). While GSNO catabolism has been observed with $\mathrm{Cu}-\mathrm{Zn}$ superoxide dismutase, GSH peroxidase, xanthine oxidase, and human carbonyl reductase 1 (CR1), the former three enzymes merely regenerate NO [summarized in Broniowska et al. (2013)], and residues critical to interaction between CR1 and GSH adducts are not conserved in plants (Bateman et al., 2008). GSNOR is therefore considered the primary catalyst for GSNO catabolism in plants.

The importance of GSNOR to plant growth, development and stress responses has been highlighted by several studies. Lowered GSNOR expression in Arabidopsis thaliana 
(Arabidopsis), resulting from a null mutation (atgsnor1-3/hot5-2) or RNAi, was correlated with higher SNO content and differential susceptibilities to pathogens (Feechan et al., 2005; Rustérucci et al., 2007). The effect of absence of GSNOR was extended by Lee et al. (2008) who described a thermotolerance defect that was rescued with NO scavengers. Other phenotypes of plants with GSNOR mutations include diminished fertility and resistance to programmed cell death induced by paraquat, an herbicide that elicits robust reactive oxygen species (ROS) production (Lee et al., 2008; Chen et al., 2009). These concomitant gains and losses of function are analogous to consequences of GSNOR inhibition in mammals, for which both enhanced carcinogenesis and abated severity of inflammatory diseases are observed (Wei et al., 2010; Sun et al., 2011; Tang et al., 2013). Such pleiotropy suggests GSNOR participates in both homeostatic maintenance and biotic and abiotic stress responses.

The evolutionary conservation of GSNOR is high (Liu et al., 2001), and although the consequences of GSNOR depletion have been described at the organismal level for Arabidopsis (Lee et al., 2008; Chen et al., 2009; Kwon et al., 2012), a molecular etiology for the GSNOR loss-of-function phenotype is lacking. Here we sought to address the issues of how GSNOR activity could be regulated and of what processes are impacted by changes in GSNO levels and, therefore, potentially regulated by nitrosation or glutathionylation of protein effectors. We searched for conserved and unique features of plant GSNOR proteins, localized GSNOR at the tissue and cellular levels, and measured global changes in the transcriptome of an atgsnor/hot5 null mutant in Arabidopsis. Our data demonstrate that most sequenced green plant genomes are predicted to encode a single copy of GSNOR characterized by a high content of positionally-conserved cysteines. GSNOR is found in the cytosol and nucleus throughout the plant, and is thus available to modulate GSNO concentration in most if not all cells. Moreover, alterations in the transcriptome of Arabidopsis homozygous for the atgsnor1-3/hot5-2 null allele exhibited dysregulated expression of pathogen response and calcium signaling genes, but higher expression of a subset of glutaredoxin (GRX)encoding genes. Together, these data suggest GSNOR facilitates multiple homeostatic and stress adaptation processes in green plants.

\section{MATERIALS AND METHODS}

\section{AMINO ACID SEOUENCE ALIGNMENTS AND ANALYSIS}

Predicted genes encoding GSNOR from green plants were retrieved from NCBI Genbank and Phytozome v9.1 (Goodstein et al., 2012) using Arabidopsis GSNOR (At5g43940, ADH2) as the tblastn query. GSNOR copy number was assessed with Phytozome and NCBI tblastn algorithms by querying predicted GSNOR- encoding genes against genomic reads from a particular plant species. Similar hits were considered duplicates if $5^{\prime}$ and $3^{\prime}$ intragenic and intron nucleotide sequences were $>99 \%$ identical. Predicted GSNORs were also aligned via ClustalW (Larkin et al., 2007) with the Arabidopsis protein most similar to GSNOR (alcohol dehydrogenase, AtADH1, Atlg77120), and sequences that cladded with AtADH1 were culled. Bacterial, metazoan, and fungal orthologs discussed in the text were uncovered through an NCBI tblastn search with E. coli, Saccharomyces cerevisiae, and human GSNOR queries, respectively. ClustalW sequence alignments were made with Jalview (Waterhouse et al., 2009). Deduced protein sequences were included in Figure 1 if transcript evidence (i.e., RNAseq and/or ESTs spanning the coding sequence) was available through Phytozome. Phylogenetic trees were drawn from aligned sequences in EvolView (Zhang et al., 2012). N-terminal targeting peptide searches were performed with Predotar (Small et al., 2004) and MITOPROT (Claros, 1995). Mitochondrial targeting peptides encoded by GSNOR $5^{\prime}$ intragenic regions were identified by six-frame translation of the first 500 base pairs upstream of the start codon using the intron splice rules for Arabidopsis (Hebsgaard et al., 1996).

\section{GSNOR STRUCTURAL ASSESSMENTS}

Crystal structures of GSNOR from tomato (4DL9), human (1MP0), and Arabidopsis (4JJI and 4GL4) were obtained from the Protein Data Bank. Graphics were made with PyMOL v 1.6 (PyMOL).

\section{PLANT MATERIAL AND GROWTH CONDITIONS}

The Arabidopsis GSNOR null mutants, hot5-2 (also known as atgsnor 1-3) (Col-0 background) and hot5-4 (WS background), have been described previously (Lee et al., 2008). Unless otherwise indicated, plants were grown in soil in growth chambers on $16 \mathrm{~h}$ days $\left(150 \mu \mathrm{Mol} \mathrm{m}{ }^{-2} \mathrm{~s}^{-1}\right.$ light intensity) and a $21 / 19^{\circ} \mathrm{C}$ day/night temperature cycle. For analysis of the number of branches produced by wild-type and mutant plants, plants were grown as above and their height and branch pattern and numbers were measured 8 weeks after germination.

\section{GENERATION AND VISUALIZATION OF GSNOR-GFP FUSIONS IN PLANTS}

Green fluorescent protein (GFP) was fused in frame $3^{\prime}$ to the Arabidopsis GSNOR genomic DNA (including $754 \mathrm{bp}$ and 180 bp of $5^{\prime}$ and $3^{\prime}$ UTR, respectively) as follows. Genomic DNA was cloned into pENTR/D TOPO (Life Technologies). GFP was obtained from pMDC83 (Curtis and Grossniklaus, 2003). HindIII and XbaI sites were added to GFP and GSNOR-encoding sequences with primers GGATGCAAGCTTAGTAAAGGAGAAG AAC and TCTCTAGATTATTTGTATAGTTCATCCATGC (GFP) and primers CGTTGTG TCCTCGATACCAGCAAGCTTGTC TCTAGATGACTATATGGGTCCTCTCTGC and GCAGAGA GGACCCATATAGTCATCTAGAGACAAGCTTGCTGGTATCG AGGAC ACAACG (GSNOR). PCR products were digested with HindIII and XbaI, purified, and ligated. The GSNOR-GFP clone was then subjected to PCR with primers GGTACCGAA TTC CTAGAGTACAACCTC and TCGAGTGCGGCCGCTAAACT AT ATGATTAG to add EcoRI and NotI sites, respectively. The PCR product was then ligated into EcoRI/NotI-digested pENTR2B. Constructs were cloned into the binary vector $\mathrm{pBIB}$ Basta-GWR (Gou et al., 2010) with LR Clonase II (Invitrogen), after which the sequence was verified.

The GSNOR-GFP translational fusion was then transformed into hot5-2 plants, and homozygous transformants were identified by Basta screening and western blots. Three independent transgenic lines were analyzed. Whole seedlings and roots were imaged 7 days after germination on minimal nutrient medium 


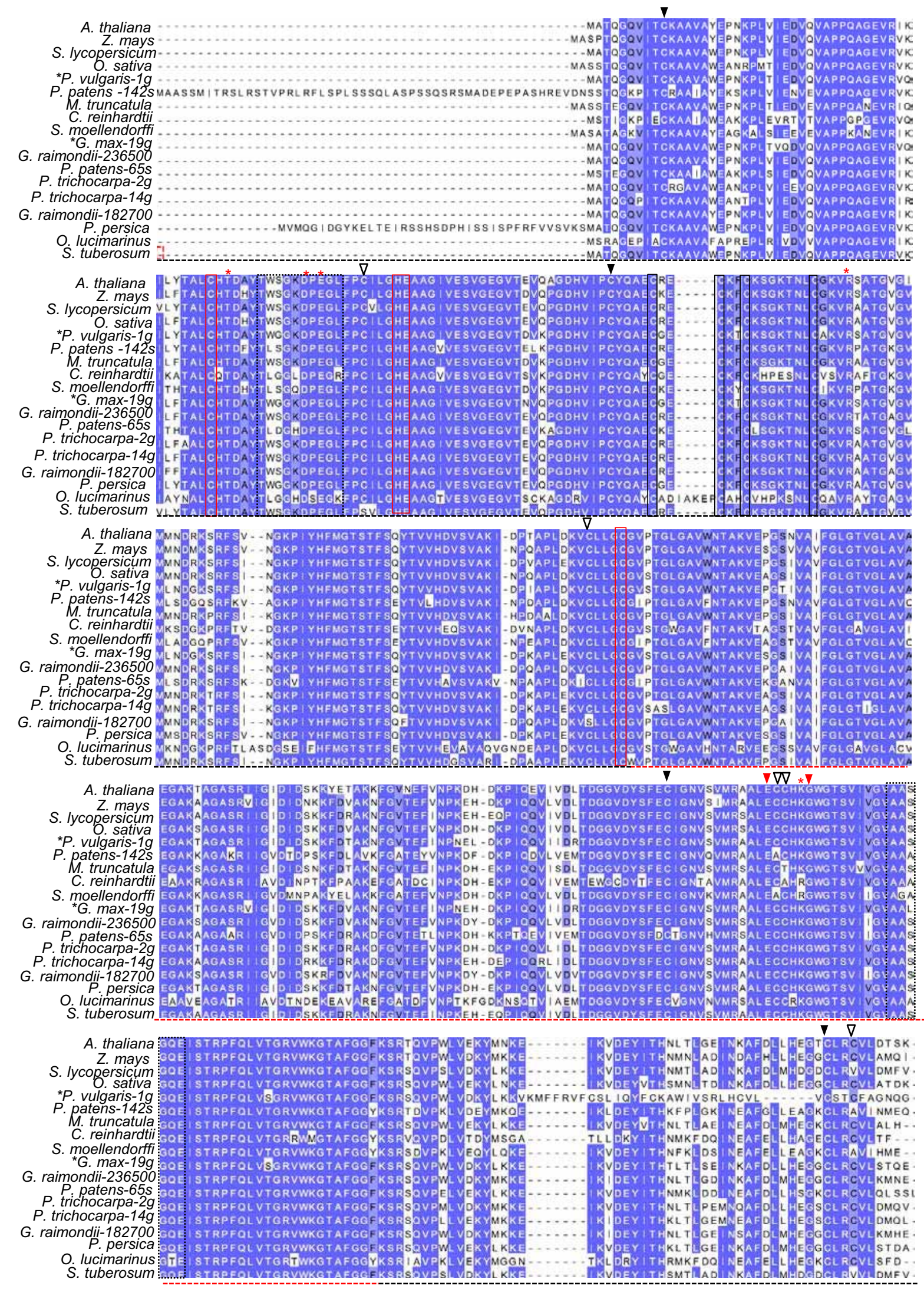

FIGURE 1 | Continued 


\section{FIGURE 1 | Continued}

Conserved features of plant GSNOR proteins. Dark blue, light blue, and uncolored residues, respectively, refer to $100 \%, \geq 75 \%$, and $<75 \%$ sequence conservation. Black and red dotted horizontal lines demarcate the catalytic and NADH-binding domains, respectively, as reported in crystal structure of tomato GSNOR (PDB code 4DL9). Residues coordinating structural and catalytic zinc atoms are outlined by solid black and red boxes, respectively. Red asterisks denote substrate-binding amino acids according to Kubienová et al. (2013). Dotted black boxes highlight flexible regions enclosing the active site. Red arrowheads $(\mathbf{v})$ indicate positions of the hot5-1 and hot5-3 missense mutations (Lee et al., 2008). Open ( $\nabla$ ) and closed ( $\mathbf{v}$ ) black arrowheads designate ex-zinc cysteines found in most and all plant sequences, respectively. Z. mays: maize. S. lycopersicum: tomato. O. sativa: rice. P. vulgaris: bean. P. patens: Physcomitrella. $M$. truncatula: Medicago. $C$. reinhardtii: Chlamydomonas. S. moellendorffii: Selaginella. G. max: soybean. G. raimondii: cotton. P. trichocarpa: poplar. P. persica: peach. O. lucimarinus: Ostreococcus. S. tuberosum: potato. S. cerevisiae: budding yeast. $H$. sapiens: human. Black asterisks: species with genomes predicted to encode additional paralogs, but only transcript-supported sequences are shown. Accession numbers can be found in Supp. Table 1 and an alignment of additional plant sequences not currently supported by transcript data is presented in Supp. Figure 1. with $0.5 \%$ sucrose. Whole flowers and reproductive structures were analyzed using stage 13 or 14 flowers as indicated in the figure legend. Images were obtained using an Olympus Fluoview FV1000 confocal microscope, with the exception of the whole seed and isolated stamens, which were imaged using conventional fluorescence microscopy with a NIKON Eclipse E800 microscope equipped with a SPOT camera (Molecular Diagnostic).

\section{MICROARRAY ANALYSIS}

Wild-type (Col) and hot5-2 Arabidopsis plants were grown on soil in a growth chamber on a $12 \mathrm{~h}$ light, $21^{\circ} \mathrm{C} / 12 \mathrm{~h}$ dark, $19^{\circ} \mathrm{C}$ cycle for 25 days after germination. Four biological replicates of wild-type and hot5-2 leaves were sampled for RNA extraction $1 \mathrm{~h}$ before the end of the light period. A two-color, dye swap hybridization was performed on a long-oligonucleotide array chip by the Galbraith lab (University of Arizona) according to published methods (Zanetti et al., 2005; Zhang et al., 2008). Data were analyzed with Robin Version 0.9.6 BETA (Lohse et al., 2010). Differentially-expressed genes were identified by two criteria: (1) change in expression greater than 2 -fold, and (2) a $t$-test $p$-value $<0.01$. Normalization of expression data, analysis using a linear model, and Benjamini and Hochberg false discovery rate correction for multiple comparisons were performed using LIMMA (Smyth and Speed, 2003).

\section{RESULTS \\ CATALYTIC AND ZINC-COORDINATING RESIDUES ARE CONSERVED IN GSNOR FROM GREEN PLANTS}

To identify potentially novel shared motifs in plant GSNORs, we employed the tblastn algorithms of Phytozome (Goodstein et al., 2012) and NCBI Gene Expression Omnibus (Altschul et al., 1997) to search for GSNOR sequences supported by transcriptional data. Eighteen unique, type III ADH-encoding cDNAs were obtained from a variety of monocots, dicots, mosses, and protists. Residues near the dimer interface of tomato GSNOR (Kubienová et al., 2013), whose mutation compromises thermotolerance in dark grown Arabidopsis seedlings (Lee et al., 2008), are notably found in all plant sequences, while a glycine that when mutated to aspartate diminishes GSNOR activity, but confers enhanced paraquat resistance (Chen et al., 2009), is present in all but two algae (Figure 1). Substrate- and NADH-enclosing clefts (Figure 1, black dotted boxes) are identical or contain conservative substitutions among moss and algal orthologs. Structural and catalytic zinc-coordinating residues (Figure 1, solid black and solid red boxes, respectively) and substrate-binding amino acids (red asterisks), as reported by Kubienová et al. (2013), are identical in all but two predicted proteins, which contain a single lysine to arginine, conservative substitution.

GSNOR is remarkably cysteine rich, with a mole percent cysteine of $3.84 \%$ for the Arabidopsis protein, compared to the $1.37 \%$ average for all proteins in the UniProtKB database (2013). Because cysteines can serve as key post-translational regulatory sites being modified by nitrosation, glutathionylation, or reversible oxidation, we analyzed the conservation of the nine extra non-zinc-coordinating cysteine residues (ex-zinc cysteines) in Arabidopsis GSNOR. Four are in all the transcript-supported plant sequences, two are substituted in one, two are substituted in two, and one differs in four organisms (Figure 1, solid and open black arrowheads), yielding an overall conservation of 93.8 $\%$ (i.e., on average, each cysteine is present in 17 of the 18 sequences). If additional plant genes are considered for which EST support is lacking, ex-zinc cysteine conservation is still $91.0 \%$ (Supp. Figure 1-see Supp. Table 1 for accession codes). Thus, the position and frequency of ex-zinc cysteines are highly conserved in plant GSNORs. We also examined the position of ex-zinc cysteines in the Arabidopsis GSNOR structure (PDB 4JJI, via (PyMOL) and found that three were solvent accessible (Cys-10, Cys-271, and Cys-370, Figures 2A-C), two of which-Cys-10 and Cys-271-are positionally conserved even in the human sequence. The structures of GSNOR from human (1MP0) and tomato (4DL9) showed similar solvent exposure of the homologous ex-zinc cysteines, suggesting these three residues may have conserved functions in regulating GSNOR activity.

\section{MOST PLANT GENOMES ENCODE ONE GSNOR PROTEIN PREDICTED TO BE FOUND IN THE CYTOSOL}

Encoded by a single gene, Arabidopsis GSNOR consists of nicotinamide cofactor-binding and catalytic domains and has two primary enzymatic activities - GSNO reductase and HMGSH dehydrogenase (Lee et al., 2008; Crotty, 2009; Kubienová et al., 2013). Interestingly, although GSNOR is found primarily as a single-copy gene in most other examined plant species (12 of 15 transcript-supported organisms, and 35 of 41 green plants analyzed), the dicots Populus trichocarpa (poplar) and Gossypium raimondii (diploid cotton) and the moss Physcomitrella patens (Physcomitrella) are predicted to have two GSNORs. Although not yet supported by transcript data, gene families are also predicted in Phaseolus vulgaris (bean), Glycine max (soybean), and Malus domestica (apple; Supp. Figure 1). Moreover, in phylogenetic trees calculated by the average distance method 

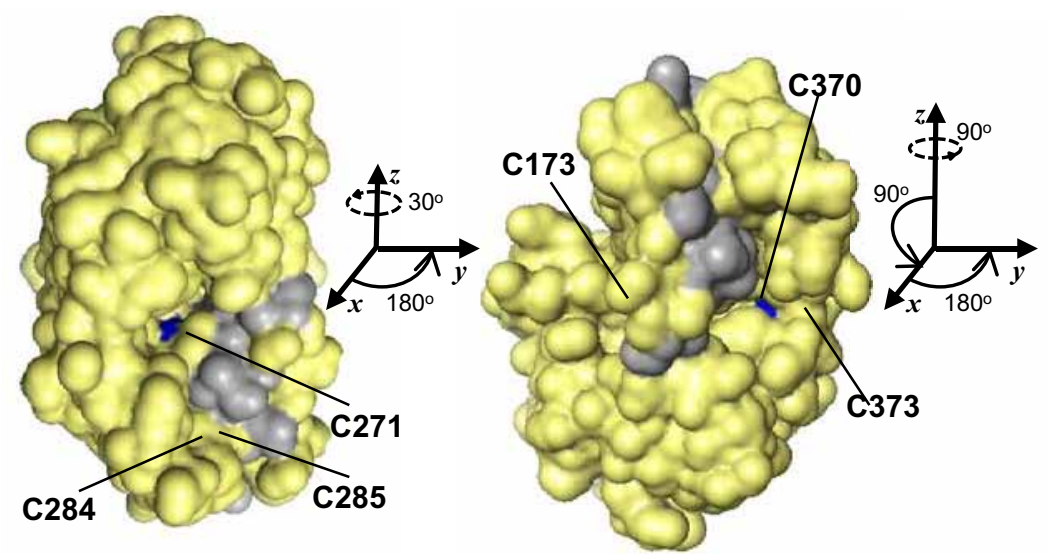

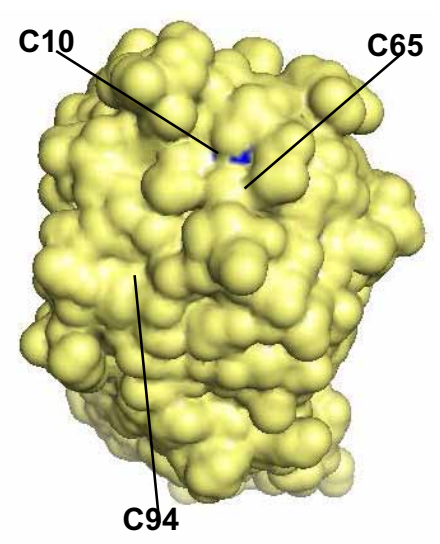

accessible surface (PDB 4JJI, $1.8 \AA$ res., $R_{\text {free }}=0.223$ ) in yellow. Solvent-accessible ex-zinc cysteines are indicated in blue, and the dimer interface in gray. Images were made in PyMOL.
FIGURE 2 | Of nine positionally-conserved ex-zinc cysteines in GSNOR three are solvent-accessible. Three orientations (rotation angles indicated) of a monomer of the Arabidopsis GSNOR dimer are shown with solvent

\section{EXPRESSION AND LOCALIZATION OF ARABIDOPSIS GSNOR}

To determine the major sites of GSNOR function, we created a translational fusion of GPF at the C-terminus of GSNOR driven by native promoter sequence and transformed this construct into the GSNOR null mutant hot5-2 (Lee et al., 2008). We carried forward three homozygous transgenic lines that express GSNOR-GFP (Figure 3A). Mutation of GSNOR results in reduced plant height and an increased number of inflorescences (Lee et al., 2008; Kwon et al., 2012). We quantified these differences in mature plants and found that while hot5-2 and hot5-4 null mutants and corresponding wild-types (Col-0 and WS, respectively) produce a similar number of first-order inflorescence stems (arising from the rosette), mutants produce two-fold more second-order branches and often produce thirdorder branches, which are not observed in wild-type plants (Figures 3B-D). All three complemented lines showed quantitative restoration of wild-type height and branching patterns (Figures 3C,D), indicating that the GFP fusion did not compromise GSNOR function. We further observed reduced trichome branching in hot5 null plants (Figures 3E,F), a previously unreported phenotype. Mutants primarily produced trichomes with only two branches, rather than the three or four typical of wild type (Figure 3F). 70\% of hot5-2 trichomes were twobranched compared to virtually zero in Col, and similar differences were detected between WS and hot5-4 (Figure 3G). The absolute number of trichomes was marginally higher in WS leaves compared with those of hot5-4, in agreement with Holzmeister et al. (2011), while trichome abundance did not differ between Col and hot5-2 (Figure 3E, lower panel), indicating the difference in trichome number must be due to a modifying gene present only in the WS background. Quantitation of trichome branching in the complemented lines showed that the GSNORGFP fusion protein restored branching to wild-type levels (Figures 3F,G).

Based on complementation of hot5 phenotypes, the GFP fusion seemed suitable to assess GSNOR localization. Whole seedlings at the cotyledon stage and flower stage 13 were 


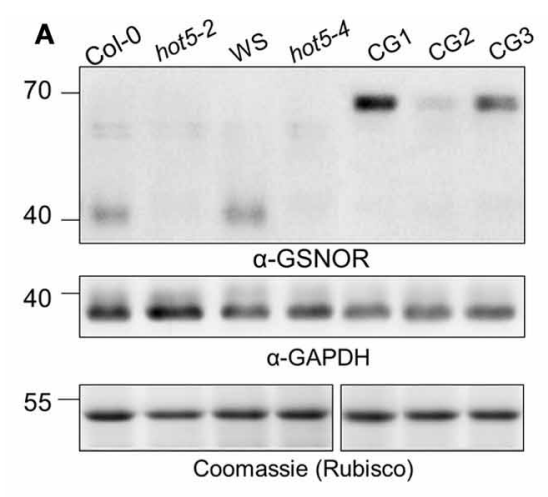

B
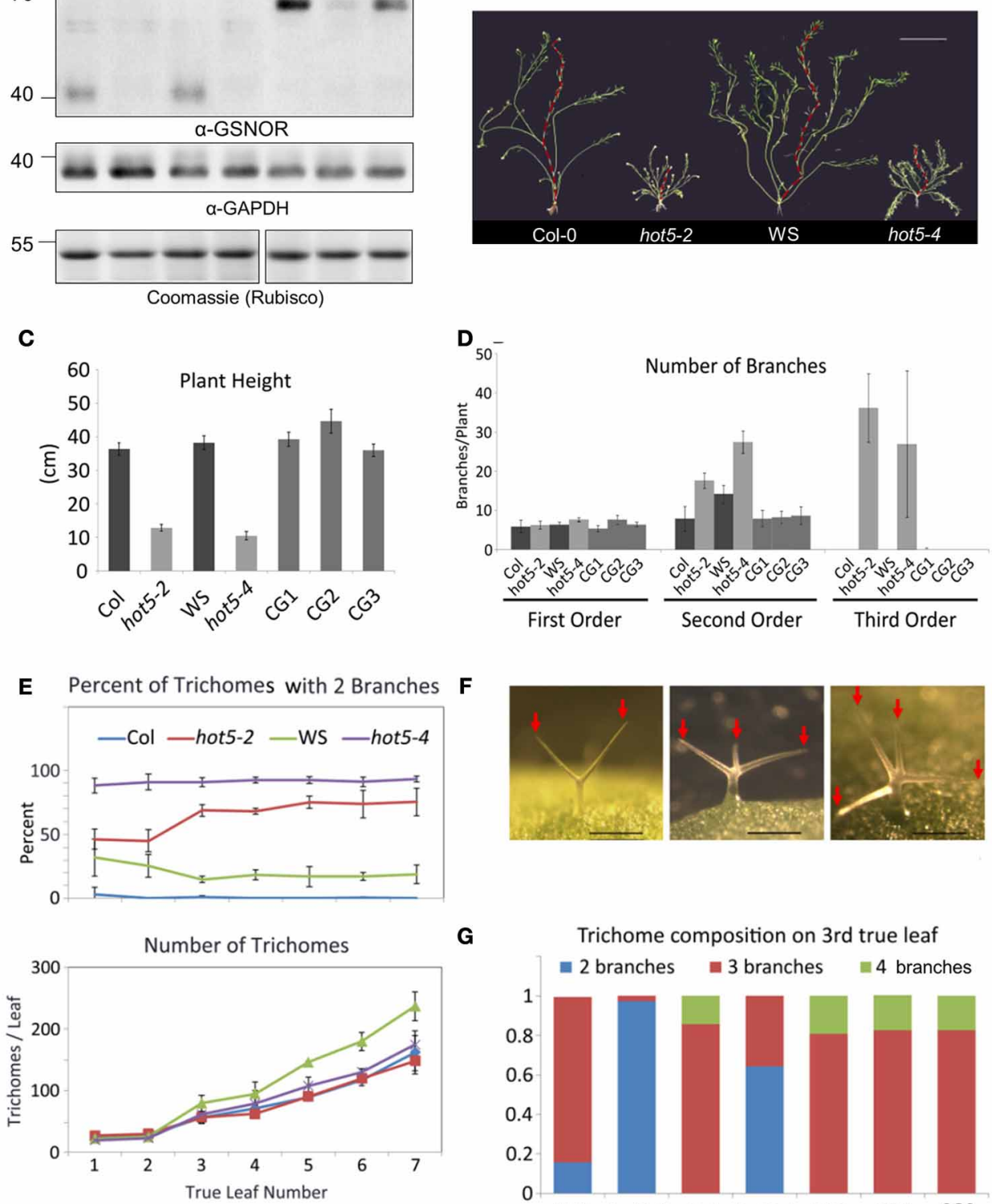

$\mathbf{F}$

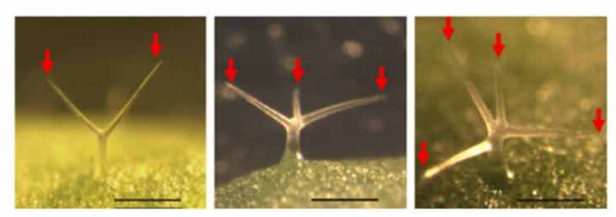

G

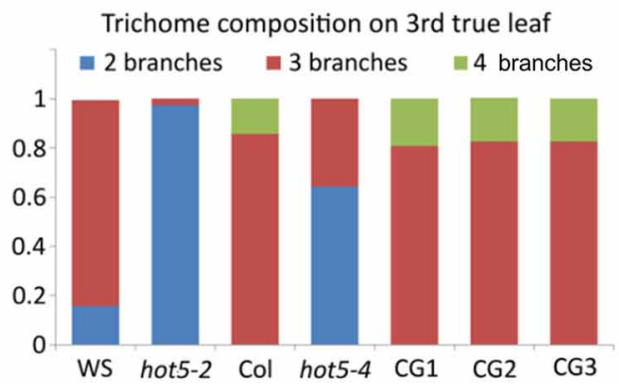

FIGURE 3 | Expression of GSNOR-GFP under its own promoter rescues multiple aspects of the hot5-2 null phenotype. (A) Immunoblots of leaf protein extracts probed with anti-Arabidopsis GSNOR antibodies (top) or anti-cytosolic GAPDH blots (middle). Rubisco large subunit (Coomassie stain, bottom). Plant genotypes are as indicated with CG1, 2, and 3 being independent homozygous T3 lines expressing GSNOR::GSNOR-GFP in the hot5-2 background. (B) Shoot systems of indicated genetic backgrounds

with primary inflorescence overlaid in red. Bar: $5 \mathrm{~cm}$. (C,D) Shorter plant height (C) and inflorescence branching order (D) are rescued to wild-type levels in CG1, 2, and 3. (E) Trichomes with reduced branching are more numerous in hot5-2 and hot5-4 rosette leaves than in respective wild-type backgrounds. Error bars: STD (F) Left to right: trichomes from hot5-2, Col, Col. Bar: $0.2 \mathrm{~mm}$. Red Arrows indicate trichome branches. (G) Trichome branching is rescued to wild-type levels in CG1,2, and 3 .

imaged to observe the tissue and subcellular localization of GSNOR-GFP (Figure 4). Fluorescence was observed throughout seedling and floral structures (Figures $4 \mathrm{~A}-\mathrm{C}$ ), which correlates with gene expression data in public databases (Toufighi et al., 2005). The seedling apical meristem and root tip exhibited notably intense fluorescence (Figures 4A,D), while cotyledon, hypocotyl, root, and petal vascular tissue signals were highest
(Figures 4A-C). Detailed observation of sections through the root tip (Figures 4E-G) show distribution in all root cell types with diffuse cytosolic and nuclear localization, but dramatic exclusion from the nucleolus. This is also evident in the elongating zone of the root, although nucleoli are considerably smaller in these cells (Figure 4H). GSNOR-GFP could also be detected in anther filaments, ovary, stigma, and petals (Figures $4 \mathbf{I}, \mathbf{J}, \mathbf{M}, \mathbf{N}, \mathbf{P}$ ) 

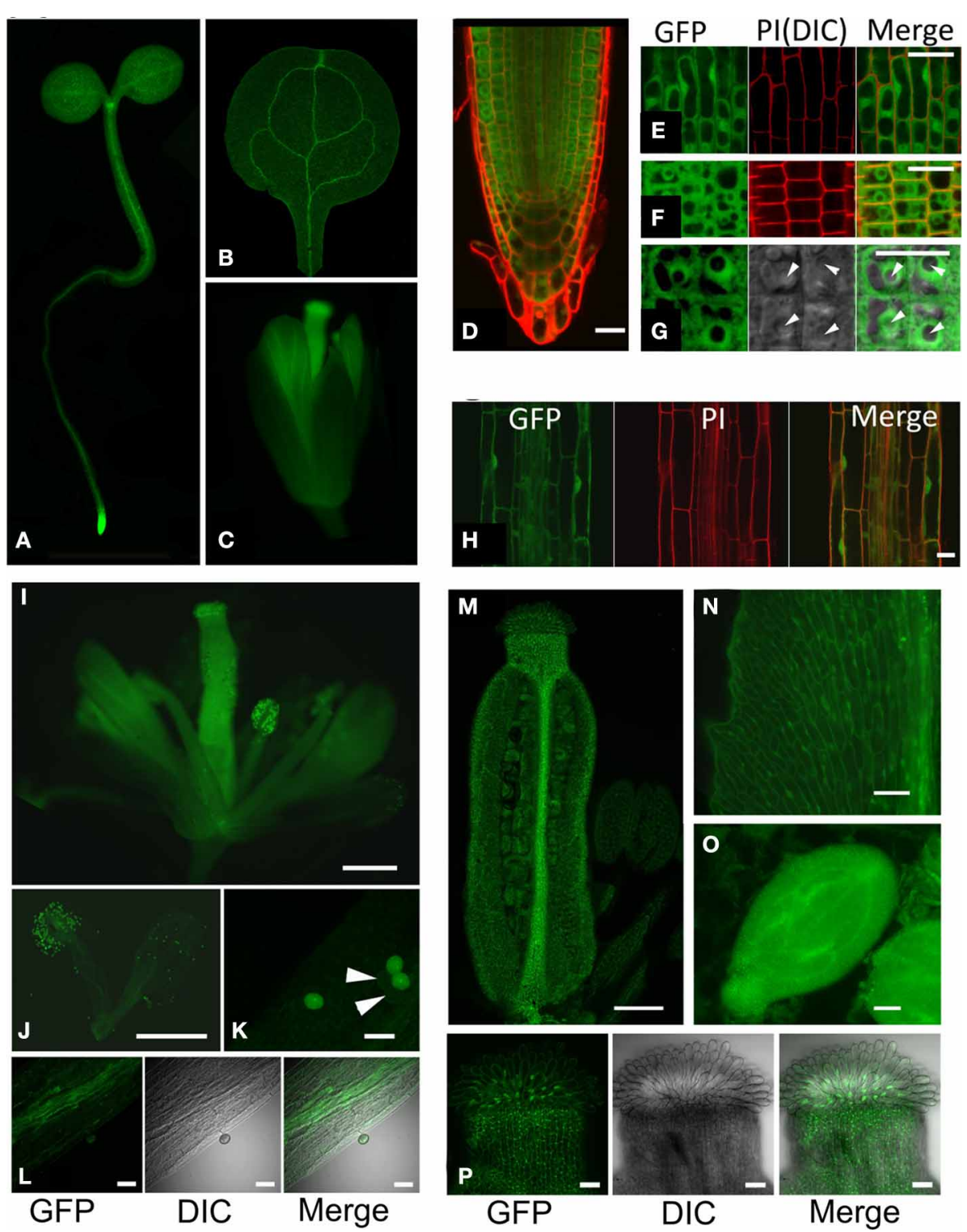

FIGURE 4 | GSNOR is expressed in various organs and developmental stages in Arabidopsis. Localization of GSNOR was observed in plants transformed with GSNOR::GSNOR-GFP in the hot5-2 background. All plants were homozygous for the GSNOR::GSNOR-GFP transgene, with the exception of those used for images in (I-K). (A) Whole seedling. (B) Cotyledon. (C) Flower, stage 13. (D) GSNOR-GFP distribution and localization in root tip cells in optical cross section through the middle of the root. (E) GSNOR-GFP localization in root epidermal cells. (F, G) Optical cross section of GSNOR-GFP localization in root cortex cells at two different magnifications. Arrowheads: nucleolus. PI, Propidium lodide staining; DIC, Differential Interference Contrast microscopy. (H) GSNOR-GFP localization in the root elongation zone. Bar: $20 \mu \mathrm{m}$ (D-H). (I-P) Localization of GSNOR-GFP in stage 14 flowers (I) stamens and petals (J) pollen (K) anther filaments (L) ovary (M) petals (N) seed at bending cotyledon embryonic stage (O) and stigma (P). Arrowheads in (K) denote hot5-2 pollen that do not express GSNOR-GFP due to segregation of the transgene in the heterozygote. Bar: $500 \mu \mathrm{m}(\mathbf{I}, \mathbf{J}) 200 \mu \mathrm{m}$ (M), or $40 \mu \mathrm{m}(\mathbf{K}, \mathbf{L}, \mathbf{N}-\mathbf{P})$. of stage 14 flowers and was particularly enriched in pollen and seed (Figures 4K,O).

\section{MULTIPLE PATHOGEN RESPONSE GENES ARE DOWNREGULATED IN hot5-2}

To assess global changes in the transcriptome due to GSNOR absence, we performed microarray analysis on 4-week leaves of hot5-2 plants grown on a $12 \mathrm{~h}$ light cycle since the leaves of Col and hot5-2 plants are most morphologically comparable at this age (Lee et al., 2008). Imposing stringent criteria of $\geq 2$-fold changes with $p \leq 0.01$ (FDR-corrected), we found 99 and 170 transcripts up- and downregulated, respectively, in hot5-2 compared to Col-0. A complete list of transcripts is provided in Supp. Table 2 categorized by pathway as curated in MAPMAN (Usadel et al., 2009). This list was used to test for enriched categories of regulated genes (Usadel et al., 2006). 
One group of enriched genes was the "Stress Response" category (Table 1). Of 19 genes in this category, 13 were downregulated "biotic stress" genes, including pathogenesis-related protein 1 (PR1, 6.9-fold), consistent with data from Feechan et al. (2005), four potential pathogen receptors (At1g59218, At3g04210, At3g50470, At3g11010), PR5, and other predicted herbivore and pathogen defense proteins. Of two upregulated "Stress" category genes, the defensin protein PDF1.2 was also reported to be upregulated in GSNOR antisense plants (Espunya et al., 2012).

These results are consistent with the involvement of NO in pathogen responses, and prompted us to employ the Arabidopsis eFP browser (Toufighi et al., 2005) and TAIR gene annotations to determine if other pathogen response-linked genes, including those in the "Not Assigned" category (the largest category with 76 genes) were also differentially regulated in hot5-2. The eFP data include response to the oomycete Phytophthora infestans, the bacterium Pseudomonas syringae (virulent and avirulent), the fungi Botrytis cinerea and Erysiphe orontii, as well as induction by non-host bacteria and several elicitors. As highlighted in Supp. Table 2, 43 additional genes that can be linked to pathogen response are down-regulated (e.g., ACD6, At4g14400; DIR1, At5g48485), making a total of 56 of 170 downregulated genes linked to pathogen response. In contrast, only 13 additional upregulated genes can be linked to pathogen response. Since $\sim 12 \%$ of Arabidopsis genes are pathogen defense-related (The Arabidopsis Initiative, 2000), we conclude the absence of GSNOR disproportionately downregulates pathogen response genes.

\section{AN UNUSUAL CLASS OF GLUTAREDOXINS ARE UPREGULATED IN hot5-2}

Given the significant interplay between GSNO levels and GSHcontrolled redox homeostasis (Staab et al., 2008), it was of interest that genes in the "Redox" category were also enriched. Intriguingly, six cytosolic ROXY-class glutaredoxins (GRXs) and an atypical chloroplast-localized thioredoxin (TRX) were upregulated in hot5-2 (Table 2 and Supp. Table 2). GRXs and TRXs are small oxidoreductases that regulate the thiol redox state of other proteins (Meyer et al., 2012). Four of the six up-regulated GRX genes are closely linked on chromosome 4 and share $91-95 \%$ identity, while two others on chromosomes 5 and 1 are $72-75 \%$ and $58-60 \%$ identical to the chromosome 4 genes, respectively, and 59\% identical to each other. All six GRXs belong to a plantspecific GRX family containing a monocysteine active site. Thus, these GRX proteins likely act as monothiol GRXs (Herrero and De La Torre-Ruiz, 2007). One cytosolic, monothiol ROXY GRX (ROXY20), which is divergent from the upregulated genes ( $40 \%$ identical), is downregulated in hot5-2. Examination of publically available expression patterns of these regulated ROXY genes (Schmid et al., 2005) indicates that during normal plant growth the hot5-2 upregulated $R O X Y$ genes have low transcript levels in stems, senescing leaves, and the shoot apex, while ROXY20 shows the opposite pattern. Thus, these GRX proteins are likely to serve different functions. Currently the redox targets of these cytosolic GRXs and the unusual chloroplast TRX are unknown, but increased expression of the corresponding genes indicates that mutation of GSNOR alters redox homeostasis and potentially the targets of these oxidoreductases.

Table 1 | Changes in "Stress Responsive" Transcripts in hot5-2 vs. wild-type.

\begin{tabular}{|c|c|c|c|c|}
\hline Gene & Protein & Stress & Fold-change (log2) & $p$-value \\
\hline \multicolumn{5}{|c|}{ UPREGULATED } \\
\hline At3g04720 & PR4 (PATHOGENESIS-RELATED GENE 4) & Biotic & 1.397 & 0.001 \\
\hline \multicolumn{5}{|c|}{ DOWNREGULATED } \\
\hline At2g14610 & PR1 (PATOGENESIS-RELATED GENE 1) & Biotic & -2.78 & 0 \\
\hline At1g73330 & DR4 (DROUGHT REPRESSED 4); peptidase inhibitor & Biotic & -1.882 & 0 \\
\hline At3g50480 & HR4 (HOMOLOG OF RPW8 4) & Biotic & -1.861 & 0.001 \\
\hline At1g75040 & PR5 (PATHOGENESIS-RELATED GENE 5) & Biotic & -1.821 & 0.001 \\
\hline At3g11010 & AtRLP34 (Receptor-like Protein 34) & Biotic & -1.8 & 0.002 \\
\hline At1g24020 & MLP423 (MajorLatexPprotein-LIKE PROTEIN 423) & Abiotic & -1.65 & 0 \\
\hline At1g59218 & Disease resistance protein (CC-NBS-LRR class) & Biotic & -1.272 & 0.001 \\
\hline At3g48080 & Lipase class 3 family protein & Biotic & -1.238 & 0.001 \\
\hline At1g72260 & THI2.1 (THIONIN 2.1) & Biotic & -1.117 & 0.001 \\
\hline At2g03720 & MRH6 (morphogenesis of root hair 6) & Abiotic & -1.083 & 0.008 \\
\hline At1g19670 & COR1 (CORONATINE-INDUCED PROTEIN 1); CHL1 (CHLOROPHYLLASE 1) & Abiotic & -1.013 & 0.001 \\
\hline
\end{tabular}

$p \leq 10^{-8}$ listed as $=0$. 
Table 2 | Changes in "Redox" Transcripts in hot5-2 vs. wild-type.

\begin{tabular}{|c|c|c|c|c|}
\hline Gene & Protein & Function/Localization & Fold-change $(\log 2)$ & $p$-value \\
\hline \multicolumn{5}{|c|}{ UPREGULATED } \\
\hline At4g15690 & Glutaredoxin S5 & Glutaredoxin; cytosolic; CxxS active site & 2.788 & $6.94 \mathrm{E}-05$ \\
\hline At4g15660 & Glutaredoxin S8 & Glutaredoxin; cytosolic; CxxS active site & 2.088 & 0 \\
\hline At4g15700 & Glutaredoxin S3 & Glutaredoxin; cytosolic; CxxS active site & 1.786 & 0 \\
\hline At5g18600 & Glutaredoxin S2 & Glutaredoxin; cytosolic; $\mathrm{CxxS}$ active site & 1.064 & 0.001 \\
\hline \multicolumn{5}{|c|}{ DOWNREGULATED } \\
\hline At5g11930 & Glutaredoxin C10 & Glutaredoxin; cytosolic; CxxS active site & -1.155 & 0.001 \\
\hline At1g20620 & CAT3 & Catalase; peroxisome; cytosol? & -1.108 & 0.002 \\
\hline
\end{tabular}

$p \leq 10^{-8}$ listed as $=0$.

\section{CHANGES IN hot5-2 GENE EXPRESSION INDICATE OTHER PROCESSES IMPACTED BY NO HOMEOSTASIS}

Another enriched category of genes that presents a consistent picture of changes in the GSNOR mutant is the "Signaling" category, in which 14 of 15 genes are down regulated and half of the downregulated genes are involved in calcium signaling, including multiple calmodulins, calmodulin-like proteins and calreticulins (Supp. Table 2). The single upregulated gene in this category is ATCP1 (CALCIUM BINDING PROTEIN 1, At5g49480), which also has sequence similarity to calmodulin. These data provide a direct link of GSNO to calcium signaling.

Although "Transcription" was not a specifically enriched category, consideration of regulated transcription factors (Supp. Table 1) shows that three of the most highly upregulated genes encode BHLH proteins involved in iron deficiency responses (BHLH100, At2g41240; BHLH039, At3g5698; BHLH038, At3g56980) (Wang et al., 2013). The potential targets of these transcription factors in leaves are unknown, although in roots they can activate genes required for iron uptake. Among downregulated genes in the transcription category, it is notable that there are three AP2/EREBP transcription factors, which are involved in ethylene responses, including the most strongly downregulated gene in this category, TINY (At5g11590; > 5-fold decreased) (Sun et al., 2008).

\section{DISCUSSION}

The pleiotropy of Arabidopsis GSNOR loss-of-function mutants indicates that enzymatic control of GSNO levels is essential for competitive viability (Lee et al., 2008; Kwon et al., 2012) (Feechan et al., 2005). In vitro, GSNOR catabolizes GSNO and HMGSH, metabolites generated from NO- and formaldehyde, respectively. Because NO can lead to the generation of reactive nitrogen species (RNS) and formaldehyde can produce ROS (Staab et al., 2008; Kubienová et al., 2013), GSNOR is likely critical to regulation of downstream physiological and pathological effects of RNS and ROS in plants. The evolutionary conservation of GSNOR in eukaryotes and bacteria, which was recognized over a decade ago (Liu et al., 2001), further indicates this enzyme has similar functions in multiple domains of life. Here we have identified conserved features of GSNOR genes and their encoded proteins in plants, determined aspects of tissue and cellular localization of GSNOR, and shown that its absence impacts expression of pathogen response, redox, and calcium signaling genes in Arabidopsis. While this work is primarily descriptive, it offers insight into the molecular consequences of GSNOR loss and therefore can serve as a prospectus for future mechanistic studies.

GSNOR was found as a single-copy gene in 35 of 41 plant species, with seven instances of recent duplication events (data not shown). De Smet et al. (2013) theorize that the preponderance of single copy genes in organisms that have undergone whole genome duplication (such as Arabidopsis) is not random, but rather indicates that these genes impair fitness when present in multiple copies, possibly due to overly-robust activity of their encoded proteins. In any event, GSNOR copy number appears to be under strong selective pressure.

All putative eukaryotic GSNORs we examined show high conservation and are unusually rich in cysteines, which are highly positionally conserved among plant orthologs (Figures 1, 2 and Supp. Figure 1). Common functions of protein cysteine residues include coordination of metal atoms (i.e., copper and zinc), covalent catalysis, extracellular adhesion, and redox sensing (Wang et al., 2012). Most of the ex-zinc cysteines in Arabidopsis GSNOR were found to be inaccessible to solvent (Figure 2; Crotty, 2009), suggesting their primary function may be structural. Intriguingly, however, three cysteines that are positionally conserved between plants and animals are solvent accessible (Figure 2, blue patches). These residues may serve as sites of post-translational regulation via, for instance, glutathionylation or S-nitrosation. Residues that bind HMGSH or coordinate zinc (Kubienová et al., 2013) were found in all predicted plant proteins (Figure 1 and Supp. Figure 1), indicating GSNO reductase and HMGSH dehydrogenase activities are probably general features of plant GSNORs. GSNORs from Arabidopsis and tomato exhibit $\sim 10$-fold higher velocity of NADH-dependent GSNO reduction than $\mathrm{NAD}^{+}$-dependent HMGSH oxidation to formylglutathione (Crotty, 2009; Kubienová et al., 2013), but the high ratio of $\mathrm{NAD}^{+}$to $\mathrm{NADH}$ in most living cells would favor the dehydrogenase reaction. This could be circumvented by cofactor recycling, wherein $\mathrm{NADH}$ produced from $\mathrm{HMGSH}$ oxidation 
is employed for GSNO reduction (Staab et al., 2008). Thus, GSNO catabolism and formaldehyde detoxification may be partially interdependent processes. This scenario is not unreasonable, as ROS and RNS are known to contribute to the formation of one another (Molassiotis and Fotopoulos, 2011). The mitochondrion is a major site of ROS production in eukaryotes. GSH and NO concentrations are reported to be high in Arabidopsis mitochondria (Wang et al., 2010; Koffler et al., 2013), and mitochondrial enzymes have been identified as nitrosation targets (Millar and Day, 1996; Palmieri et al., 2010). There is no transcriptional evidence for mitochondrion-localized GSNORs apart from one Physcomitrella homolog (Figure 1), but the existence of cryptic target peptide-encoding sequences in frame with GSNORs from diverse taxa (data not shown) underscores possible mitochondrial localization in a common evolutionary ancestor.

Visualization of GSNOR-GFP provided clear evidence for cytosolic and nuclear localization of this protein throughout the plant, with potentially higher concentrations in vascular tissues and very noticeable exclusion from the nucleolus. Though GSNOR lacks a nuclear localization signal, it may be transported in association with another protein. We saw no evidence of GSNOR-GFP in mitochondria, but the upstream region with potential to encode a mitochondrial targeting peptide was not included in our construct. Our results of organ and tissue localization do not fully agree with a previous report from Espunya et al. (2006); while they found high levels of activity in roots, no activity was detected in the hypocotyl or cotyledons, which show significant fluorescence in our study. Consistent with our observations, their immunocytochemistry showed GSNOR in all cell types of the root meristematic zone. However, the inner cortex of the root elongation zone appeared to lack protein when localized by immunocytochemistry, in contrast to our observations. Overall, GSNOR appears to function in essentially all plant cell types. These protein data are augmented by publically available transcript analysis in Arabidopsis, which show virtually ubiquitous expression of GSNOR mRNA, further supporting the role of this enzyme in multiple plant processes.

A well-documented feature of GSNOR null Arabidopsis is a multi-branching phenotype (Lee et al., 2008), which has been suggested to arise from impact on auxin transport and function, and/or cytokinin signaling (Kwon et al., 2012). Replacement of wild-type GSNOR with GSNOR C-terminally fused to GFP produced plants with quantitatively normal branching patterns, indicating GSNOR-GFP effectively functions like the wild-type protein in processes required for normal branching. The GSNORGFP transgene also rescued reduced trichome branching, a previously unrecognized phenotype of the null mutant. Arabidopsis trichomes are single cells, so reduced branching results from alteration of cell morphogenesis, which involves a wide range of basic processes (Hülskamp, 2000). How absence of GSNOR reduces trichome branches is not known, but notably, Arabidopsis trichomes have two to four times the concentration of GSH compared to other epidermal cells (Gutiérrez-Alcalá et al., 2000). High GSH may increase the deleterious effects of the absence of GSNOR specifically in these cells. GSNOR-GPF also rescued this phenotype, implying a role for NO homeostasis in basic cell morphological processes. The pleiotropy of the GSNOR loss-of-function phenotype suggests enhanced nitrosation of one or more proteins interferes with their normal physiological functions. However, there are only a few known in vivo targets of nitrosation in plants: the Arabidopsis cytokinin signal relay kinase AHP1 is negatively regulated via nitrosation of a conserved cysteine (Feng et al., 2013), while nitrosation of the $\mathrm{F}$ box protein TIR1 has been proposed to modulate auxin signaling (Terrile et al., 2012). Thus, the strong morphological phenotypes of hot5-2 and hot5-4 plants may reflect uncoordinated cytokinin and auxin crosstalk caused by aberrant nitrosation of TIR1 and AHP1. Observed changes in pathogen sensitivity may have a similar etiology. Indeed, salicylic acid (SA) signaling has been shown to be downreguated by GSNO-mediated nitrosation of NPR1 (Tada et al., 2008). Further work with the GSNOR null mutant will be required to determine any alterations in nitrosated or glutathionylated targets.

Comparison of transcript levels between a GSNOR null mutant and wild type Arabidopsis on microarrays revealed an enrichment in transcripts involved in pathogen responses, redox regulation, and calcium signaling, as well as upregulation of transcription factors involved in iron responses and downregulation of ethylene responsive transcription factors. The extensive downregulation of pathogen defense related and responsive genes supports the observations of Feechan et al. (2005), who reported the null mutant had reduced $\mathrm{R}$ gene mediated, basal and non-host resistance to pathogens. While the Arabidopsis homolog of human CR1 was not transcriptionally higher in the hot5-2 mutant, as might be expected if it provided compensatory activity, GRXs and a TRX were notably upregulated. TRXs and GRXs can mediate denitrosation and deglutathionylation, respectively (Benhar et al., 2009; Zaffagnini et al., 2013), such that they could function to reverse an increase in these modifications due to excess GSNO in the mutant. Although the contribution of TRXs and GRXs to the acclimation of GSNOR-deficient plants to nitrosative stress has not been assessed, these microarray data strongly implicate the involvement of GRXs in plant SNO homeostasis. The microarray data also support proposed linkages between NO and calcium signaling (Courtois et al., 2008), as five calmodulins or calcium binding proteins are downregulated and one is upregulated in hot5-2.

We also compared the results of our microarrays with previous studies aimed at identifying $\mathrm{NO}$ regulated genes that used NO donors or NO synthase inhibitors to induce changes in gene expression in Arabidopsis (Parani et al., 2004; Besson-Bard et al., 2009). We found very limited overlap with potentially NO-regulated transcripts. For genes induced by application of 0.1 or $1.0 \mathrm{mM}$ SNP (Parani et al., 2004), only four genes were also upregulated in hot5-2: the calmodulin-like ATCP1 (At5g49480), WRKY40 (Atlg80840), a UDP glucosyl transferase (At1g05560) and a 67 amino acid unknown (At4g27654). Compared to transcripts regulated by a NOS inhibitor, in which downregulated genes were proposed to be normal targets of NO, again only four transcripts behaved similarly in our experiments (DIN10, At5g20250; XTR8,At3g44990; 
LTP1,At2g45780; unknown, At3g56360). We may see little overlap with these prior investigations because they examined short-term manipulation of NO levels, whereas GSNO and NO are chronically deregulated in hot5-2. In any event, changes in gene expression in the absence of GSNOR reflect a long-term metabolic adjustment required to cope with excess GSNO and the pathways it normally regulates.

GSNOR clearly plays a role in biotic stress adaptation, but how a GSNOR-DOD fusion such as that identified in a homolog from apple (Supp. Figure 1) would function in vivo is not clear, and this may be an artifact of early phase sequencing. Using the Toronto Bio-Analytic Resource (BAR) Arabidopsis gene expression data compendium (Toufighi et al., 2005), it was revealed that GSNOR transcription strongly correlated with NINJA $\left[r^{2}=0.62\right.$, a jasmonic acid (JA) response corepressor] and PMR5 $\left(r^{2}=0.59\right.$, a protein whose absence affords greater resistance to biotrophic fungi that cause powdery mildew). Both PMR5 and NINJA negatively regulate pathogen-induced defense signaling-PMR5 contributes to JA-independent fungal disease susceptibility (Vogel et al., 2004), while NINJA activity is curtailed following JA-induced, COI1-dependent proteasomal degradation of JAZ repressor proteins (Pauwels et al., 2010; Sheard et al., 2010). While the association of these pathogen response genes with GSNOR is only correlative, it can be inferred that GSNOR might also work to dampen biotic stress responses in the absence of elicitation. This would explain why GSNOR over-expression and RNAi-mediated knockdown served to respectively diminish and enhance systemic acquired tolerance and basal tolerance to a $P$. syringae and Peronospora parasitica (Rustérucci et al., 2007). This also harmonizes well with the observation that GSNOR positively affects SA signaling (Feechan et al., 2005), since JA and SA operate antagonistically to one another.

In summary, GSNOR appears to be a ubiquitously-expressed, cytosol-localized protein that regulates shoot morphology, pathogen defense responses, and NO homeostasis. Aberrant nitrosation of auxin, cytokinin, and SA response regulator proteins, among others, likely contribute to aspects of hot 5 null mutant phenotypes. Diminished branching in GSH-rich trichome cells further underscores the role of GSNOR in maintaining the cellular reduction potential, and its conserved, solvent-accessible cysteines may function as NO sinks or serve a regulatory role. The upregulation of transcripts of a class of GRXs is a particularly promising discovery, as some GRXs and TRXs catalyze deglutathionylation and denitrosation. Understanding how GRXs may compensate for loss of GSNOR and how GSNOR activity may be regulated through its conserved, solvent accessible cysteine residues will help to clarify the role of GSNOR in plant biology.

\section{ACKNOWLEDGMENTS}

We thank Debbie Raynes for preparing the GSNOR-GFP translational fusion vector and Dr. Fionn McLoughlin for help with confocal microscopy and discussion of the data. This research was supported by USDA NIFA grant 2008-35318-04551 and a Massachusetts Life Sciences Center (MLSC) New Faculty research award to Elizabeth Vierling.

\section{SUPPLEMENTARY MATERIAL}

The Supplementary Material for this article can be found online at: http://www.frontiersin.org/journal/10.3389/fpls.2013.00430/ abstract

\section{REFERENCES}

The Arabidopsis Initiative. (2000). Analysis of the genome sequence of the flowering plant Arabidopsis thaliana. Nature 408, 796-815. doi: 10.1038/35048692

(2013). UniProtKB/Swiss-Prot protein knowledgebase release 2013_07 statistics. Available online at: http://web.expasy.org/docs/relnotes/relstat.html

Achkor, H., Díaz, M., Fernández, M. R., Biosca, J. A., Parés, X., and Martínez, M. C. (2003). Enhanced formaldehyde detoxification by overexpression of glutathione-dependent formaldehyde dehydrogenase from Arabidopsis. Plant Physiol. 132, 2248-2255. doi: 10.1104/pp.103.022277

Altschul, S. F., Madden, T. L., Schaffer, A. A., Zhang, J., Zhang, Z., Miller, W., et al. (1997). Gapped BLAST and PSI-BLAST: a new generation of protein database search programs. Nucleic Acids Res. 25, 3389-3402. doi: 10.1093/nar/ 25.17.3389

Bateman, R. L., Rauh, D., Tavshanjian, B., and Shokat, K. M. (2008). Human carbonyl reductase 1 is an S-nitrosoglutathione reductase. J. Biol. Chem. 283, 35756-35762. doi: 10.1074/jbc.M807125200

Benhar, M., Forrester, M. T., and Stamler, J. S. (2009). Protein denitrosylation: enzymatic mechanisms and cellular functions. Nat. Rev. Mol. Cell Biol. 10, 721-732. doi: $10.1038 / \mathrm{nrm} 2764$

Besson-Bard, A., Astier, J., Rasul, S., Wawer, I., Dubreuil-Maurizi, C., Jeandroz, S., et al. (2009). Current view of nitric oxide-responsive genes in plants. Plant Sci. 177, 302-309. doi: 10.1016/j.plantsci.2009.06.006

Broniowska, K. A., Diers, A. R., and Hogg, N. (2013). S-nitrosoglutathione. Biochim. Biophys. Acta 1830, 3173-3181. doi: 10.1016/j.bbagen.2013.02.004

Chen, R., Sun, S., Wang, C., Li, Y., Liang, Y., An, F., et al. (2009). The Arabidopsis PARAQUAT RESISTANT2 gene encodes an S-nitrosoglutathione reductase that is a key regulator of cell death. Cell Res. 19, 1377-1387. doi: 10.1038/cr.2009.117

Claros, M. G. (1995). MitoProt, a Macintosh application for studying mitochondrial proteins. Comput. Appl. Biosci. 11, 441-447. doi: 10.1093/bioinformatics/11.4.441

Corpas, F. J., Alché, J. D. D., and Barroso, J. B. (2013). Current overview of S-nitrosoglutathione (GSNO) in higher plants. Front. Plant Sci. 4:126. doi: $10.3389 /$ fpls.2013.00126

Courtois, C., Besson, A., Dahan, J., Bourque, S., Dobrowolska, G., Pugin, A., et al. (2008). Nitric oxide signalling in plants: interplays with $\mathrm{Ca} 2+$ and protein kinases. J. Exp. Bot. 59, 155-163. doi: 10.1093/jxb/erm197

Crotty, J. (2009). Crystal Structures and Kinetics of S-Nitrosoglutathione Reductase from Arabidopsis thaliana and Human. Ph.D. dissertation, The University of Arizona, Tuscon, AZ.

Curtis, M. D., and Grossniklaus, U. (2003). A gateway cloning vector set for highthroughput functional analysis of genes in planta. Plant Physiol. 133, 462-469. doi: 10.1104/pp.103.027979

De Smet, R., Adams, K. L., Vandepoele, K., Van Montagu, M. C. E., Maere, S., and Van De Peer, Y. (2013). Convergent gene loss following gene and genome duplications creates single-copy families in flowering plants. Proc. Natl. Acad. Sci. U.S.A. 110, 2898-2903. doi: 10.1073/pnas.1300127110

Espunya, M. C., De Michele, R., Gómez-Cadenas, A., and Martínez, M. C. (2012). S-Nitrosoglutathione is a component of wound- and salicylic acid-induced systemic responses in Arabidopsis thaliana. J. Exp. Bot. 63, 3219-3227. doi: $10.1093 / \mathrm{jxb} / \mathrm{ers} 043$

Espunya, M. C., Diaz, M., Moreno-Romero, J., and Martinez, M. C. (2006). Modification of intracellular levels of glutathione-dependent formaldehyde dehydrogenase alters glutathione homeostasis and root development. Plant Cell Environ. 29, 1002-1011. doi: 10.1111/j.1365-3040.2006.01497.x

Feechan, A., Kwon, E., Yun, B.-W., Wang, Y., Pallas, J. A., and Loake, G. J. (2005). A central role for S-nitrosothiols in plant disease resistance. Proc. Natl. Acad. Sci. U.S.A. 102, 8054-8059. doi: 10.1073/pnas.0501456102

Feng, J., Wang, C., Chen, Q., Chen, H., Ren, B., Li, X., et al. (2013). S-nitrosylation of phosphotransfer proteins represses cytokinin signaling. Nat. Commun. 4, 1529. doi: $10.1038 /$ ncomms 2541

Floryszak-Wieczorek, J., Arasimowicz, M., Milczarek, G., Jelen, H., and Jackowiak, H. (2007). Only an early nitric oxide burst and the following wave of secondary nitric oxide generation enhanced effective defence responses of pelargonium 
to a necrotrophic pathogen. New Phytol. 175, 718-730. doi: 10.1111/j.14698137.2007.02142.x

Georgiev, V. G., Weber, J., Kneschke, E. M., Denev, P. N., Bley, T., and Pavlov, A. I. (2010). Antioxidant activity and phenolic content of betalain extracts from intact plants and hairy root cultures of the red beetroot Beta vulgaris cv. Detroit dark red. Plant Foods Hum. Nutr. 65, 105-111. doi: 10.1007/s11130-010-0156-6

Giustarini, D., Milzani, A., Aldini, G., Carini, M., Rossi, R., and DalleDonne, I. (2005). S-nitrosation versus S-glutathionylation of protein sulfhydryl groups by S-nitrosoglutathione. Antioxid. Redox Signal. 7, 930-939. doi: 10.1089/ars.2005.7.930

Goodstein, D. M., Shu, S., Howson, R., Neupane, R., Hayes, R. D., Fazo, J., et al (2012). Phytozome: a comparative platform for green plant genomics. Nucleic Acids Res. 40, D1178-D1186. doi: 10.1093/nar/gkr944

Gou, X., He, K., Yang, H., Yuan, T., Lin, H., Clouse, S. D., et al. (2010). Genomewide cloning and sequence analysis of leucine-rich repeat receptor-like protein kinase genes in Arabidopsis thaliana. BMC Genomics 11:19. doi: 10.1186/14712164-11-19

Gutiérrez-Alcalá, G., Gotor, C., Meyer, A. J., Fricker, M., Vega, J. M., and Romero, L. C. (2000). Glutathione biosynthesis in Arabidopsis trichome cells. Proc. Natl. Acad. Sci. U.S.A. 97, 11108-11113. doi: 10.1073/pnas.190334497

Hebsgaard, S. M., Korning, P. G., Tolstrup, N., Engelbrecht, J., Rouze, P., and Brunak, S. (1996). Splice site prediction in Arabidopsis thaliana pre-mRNA by combining local and global sequence information. Nucleic Acids Res. 24, 3439-3452. doi: 10.1093/nar/24.17.3439

Herrero, E., and De La Torre-Ruiz, M. A. (2007). Monothiol glutaredoxins: a common domain for multiple functions. Cell. Mol. Life Sci. 64, 1518-1530. doi: 10.1007/s00018-007-6554-8

Holzmeister, C., Frohlich, A., Sarioglu, H., Bauer, N., Durner, J., and Lindermayr, C. (2011). Proteomic analysis of defense response of wildtype Arabidopsis thaliana and plants with impaired NO- homeostasis. Proteomics 11, 1664-1683. doi: $10.1002 / \mathrm{pmic} .201000652$

Hülskamp, M. (2000). Cell morphogenesis: How plants split hairs. Curr. Biol. 10, R308-R310. doi: 10.1016/S0960-9822(00)00437-1

Jensen, D. E., Belka, G. K., and Du Bois, G. C. (1998). S-Nitrosoglutathione is a substrate for rat alcohol dehydrogenase class III isoenzyme. Biochem. J. 331, 659-668.

Koffler, B. E., Bloem, E., Zellnig, G., and Zechmann, B. (2013). High resolution imaging of subcellular glutathione concentrations by quantitative immunoelectron microscopy in different leaf areas of Arabidopsis. Micron 45, 119-128. doi: 10.1016/j.micron.2012.11.006

Kubienová, L., Kopecny, D., Tylichova, M., Briozzo, P., Skopalova, J., Sebela, M., et al. (2013). Structural and functional characterization of a plant S-nitrosoglutathione reductase from Solanum lycopersicum. Biochimie 95, 889-902. doi: 10.1016/j.biochi.2012.12.009

Kwon, E., Feechan, A., Yun, B. W., Hwang, B. H., Pallas, J. A., Kang, J. G., et al. (2012). AtGSNOR1 function is required for multiple developmental programs in Arabidopsis. Planta 236, 887-900. doi: 10.1007/s00425-012-1697-8

Larkin, M. A., Blackshields, G., Brown, N. P., Chenna, R., McGettigan, P. A., McWilliam, H., et al. (2007). Clustal W and Clustal X version 2.0. Bioinformatics 23, 2947-2948. doi: 10.1093/bioinformatics/btm404

Lee, U., Wie, C., Fernandez, B. O., Feelisch, M., and Vierling, E. (2008). Modulation of nitrosative stress by S-nitrosoglutathione reductase is critical for thermotolerance and plant growth in Arabidopsis. Plant Cell 20, 786-802. doi: 10.1105/tpc.107.052647

Liu, L., Hausladen, A., Zeng, M., Que, L., Heitman, J., and Stamler, J. S. (2001). A metabolic enzyme for S-nitrosothiol conserved from bacteria to humans. Nature 410, 490-494. doi: 10.1038/35068596

Lohse, M., Nunes-Nesi, A., Kruger, P., Nagel, A., Hannemann, J., Giorgi, F. M. et al. (2010). Robin: an intuitive wizard application for R-based expression microarray quality assessment and analysis. Plant Physiol. 153, 642-651. doi: 10.1104/pp.109.152553

Lozano-Juste, J., and Leon, J. (2011). Nitric oxide regulates DELLA content and PIF expression to promote photomorphogenesis in Arabidopsis. Plant Physiol. 156, 1410-1423. doi: 10.1104/pp.111.177741

Meyer, Y., Belin, C., Delorme-Hinoux, V., Reichheld, J. P., and Riondet, C. (2012). Thioredoxin and glutaredoxin systems in plants: molecular mechanisms, crosstalks, and functional significance. Antioxid. Redox Signal. 17, 1124-1160. doi: 10.1089/ars.2011.4327
Millar, A. H., and Day, D. A. (1996). Nitric oxide inhibits the cytochrome oxidase but not the alternative oxidase of plant mitochondria. FEBS Lett. 398, 155-158. doi: 10.1016/S0014-5793(96)01230-6

Molassiotis, A., and Fotopoulos, V. (2011). Oxidative and nitrosative signaling in plants: two branches in the same tree? Plant Signal. Behav. 6, 210-214. doi: 10.4161/psb.6.2.14878

Neill, S. J., Desikan, R., Clarke, A., and Hancock, J. T. (2002). Nitric oxide is a novel component of abscisic acid signaling in stomatal guard cells. Plant Physiol. 128, 13-16. doi: 10.1104/pp.010707

Palmieri, M. C., Lindermayr, C., Bauwe, H., Steinhauser, C., and Durner, J. (2010). Regulation of plant glycine decarboxylase by s-nitrosylation and glutathionylation. Plant Physiol. 152, 1514-1528. doi: 10.1104/pp.109.152579

Parani, M., Rudrabhatla, S., Myers, R., Weirich, H., Smith, B., Leaman, D. W., et al. (2004). Microarray analysis of nitric oxide responsive transcripts in Arabidopsis. Plant Biotechnol. J. 2, 359-366. doi: 10.1111/j.1467-7652.2004.00085.x

Pauwels, L., Barbero, G. F., Geerinck, J., Tilleman, S., Grunewald, W., Perez, A. C., et al. (2010). NINJA connects the co-repressor TOPLESS to jasmonate signalling. Nature 464, 788-791. doi: 10.1038/nature08854

Pymol The PyMol Molecular Graphics System, Version 1.6 Schrodinger, LLC. Available online at: http://www.pymol.org/citing

Romeo, A. A., Capobianco, J. A., and English, A. M. (2002). Heme nitrosylation of deoxyhemoglobin by s-nitrosoglutathione requires copper. J. Biol. Chem. 277, 24135-24141. doi: 10.1074/jbc.M202221200

Rustérucci, C., Espunya, M. C., Díaz, M., Chabannes, M., and Martínez, M. C. (2007). S-Nitrosoglutathione reductase affords protection against pathogens in arabidopsis, both locally and systemically. Plant Physiol. 143, 1282-1292. doi: 10.1104/pp.106.091686

Schmid, M., Davison, T. S., Henz, S. R., Pape, U. J., Demar, M., Vingron, M., et al. (2005). A gene expression map of Arabidopsis thaliana development. Nat. Genet. 37, 501-506. doi: 10.1038/ng1543

Sheard, L. B., Tan, X., Mao, H., Withers, J., Ben-Nissan, G., Hinds, T. R., et al. (2010). Jasmonate perception by inositol-phosphate-potentiated COI1-JAZ coreceptor. Nature 468, 400-405. doi: 10.1038/nature09430

Small, I., Peeters, N., Legeai, F., and Lurin, C. (2004). Predotar: a tool for rapidly screening proteomes for N-terminal targeting sequences. Proteomics 4, 1581-1590. doi: 10.1002/pmic.200300776

Smyth, G. K., and Speed, T. (2003). Normalization of cDNA microarray data. Methods 31, 265-273. doi: 10.1016/S1046-2023(03)00155-5

Staab, C. A., Ålander, J., Brandt, M., Lengqvist, J., Morgenstern, R., Grafström, R. C., et al. (2008). Reduction of S-nitrosoglutathione by alcohol dehydrogenase 3 is facilitated by substrate alcohols via direct cofactor recycling and leads to GSH-controlled formation of glutathione transferase inhibitors. Biochem. J. 413, 493-504. doi: 10.1042/BJ20071666

Sun, S., Yu, J. P., Chen, F., Zhao, T. J., Fang, X. H., Li, Y. Q., et al. (2008). TINY, a dehydration-responsive element (DRE)-binding protein-like transcription factor connecting the DRE- and ethylene-responsive element-mediated signaling pathways in Arabidopsis. J. Biol. Chem. 283, 6261-6271. doi: 10.1074/jbc.M706800200

Sun, X., Wasley, J. W. F., Qiu, J., Blonder, J. P., Stout, A. M., Green, L. S., et al. (2011). Discovery of S-nitrosoglutathione reductase inhibitors: potential agents for the treatment of asthma and other inflammatory diseases. ACS Med. Chem. Lett. 2 402-406. doi: 10.1021/ml200045s

Tada, Y., Spoel, S. H., Pajerowska-Mukhtar, K., Mou, Z., Song, J., Wang, C., et al. (2008). Plant immunity requires conformational charges of NPR1 via S-nitrosylation and thioredoxins. Science 321, 952-956. doi: 10.1126/science. 1156970

Tang, C.-H., Wei, W., Hanes, M. A., and Liu, L. (2013). Increased hepatocarcinogenesis from GSNOR deficiency in mice is prevented by pharmacological inhibition of iNOS. Cancer Res. 73, 2897-2904. doi: 10.1158/0008-5472.can12-3980

Terrile, M. C., Paris, R., Calderon-Villalobos, L. I., Iglesias, M. J., Lamattina, L., Estelle, M., et al. (2012). Nitric oxide influences auxin signaling through S-nitrosylation of the Arabidopsis TRANSPORT INHIBITOR RESPONSE 1 auxin receptor. Plant J. 70, 492-500. doi: 10.1111/j.1365-313X. 2011.04885.x

Toufighi, K., Brady, S. M., Austin, R., Ly, E., and Provart, N. J. (2005). The botany array resource: e-northerns, expression angling, and promoter analyses. Plant J. 43, 153-163. doi: 10.1111/j.1365-313X.2005.02437.x 
Usadel, B., Nagel, A., Steinhauser, D., Gibon, Y., Blasing, O. E., Redestig, H., et al. (2006). PageMan: an interactive ontology tool to generate, display, and annotate overview graphs for profiling experiments. BMC Bioinformatics 7:535. doi: 10.1186/1471-2105-7-535

Usadel, B., Poree, F., Nagel, A., Lohse, M., Czedik-Eysenberg, A., and Stitt, M. (2009). A guide to using MapMan to visualize and compare Omics data in plants: a case study in the crop species, Maize. Plant Cell Environ. 32, 1211-1229. doi: 10.1111/j.1365-3040.2009.01978.x

Vogel, J. P., Raab, T. K., Somerville, C. R., and Somerville, S. C. (2004). Mutations in PMR5 result in powdery mildew resistance and altered cell wall composition. Plant J. 40, 968-978. doi: 10.1111/j.1365-313X.2004.02264.x

Wang, N., Cui, Y., Liu, Y., Fan, H., Du, J., Huang, Z., et al. (2013). Requirement and functional redundancy of Ib subgroup bHLH proteins for iron deficiency responses and uptake in Arabidopsis thaliana. Mol. Plant 6, 503-513. doi: $10.1093 / \mathrm{mp} / \mathrm{sss} 089$

Wang, X., Li, J., Liu, J., He, W., and Bi, Y. (2010). Nitric oxide increases mitochondrial respiration in a cGMP-dependent manner in the callus from Arabidopsis thaliana. Nitric Oxide 23, 242-250. doi: 10.1016/j.niox.2010. 07.004

Wang, Y., Yang, J., and Yi, J. (2012). Redox sensing by proteins: oxidative modifications on cysteines and the consequent events. Antioxid. Redox Signal. 16, 649-657. doi: 10.1089/ars.2011.4313

Waterhouse, A. M., Procter, J. B., Martin, D. M. A., Clamp, M., and Barton, G. J. (2009). Jalview Version 2-a multiple sequence alignment editor and analysis workbench. Bioinformatics 25, 1189-1191. doi: 10.1093/bioinformatics/ btp033

Wei, W., Li, B., Hanes, M. A., Kakar, S., Chen, X., and Liu, L. (2010). S-nitrosylation from GSNOR deficiency impairs DNA repair and promotes hepatocarcinogenesis. Sci. Trans. Med. 2, 19ra13. doi: 10.1126/scitranslmed.3000328

Zaffagnini, M., Morisse, S., Bedhomme, M., Marchand, C. H., Festa, M., Rouhier, N., et al. (2013). Mechanisms of nitrosylation and denitrosylation of cytoplasmic glyceraldehyde-3-phosphate dehydrogenase from
Arabidopsis thaliana. J. Biol. Chem. 288, 22777-22789. doi: 10.1074/jbc. M113.475467

Zanetti, M. E., Chang, I. F., Gong, F., Galbraith, D. W., and Bailey-Serres, J. (2005). Immunopurification of polyribosomal complexes of Arabidopsis for global analysis of gene expression. Plant Physiol. 138, 624-635. doi: 10.1104/pp.105.059477

Zhang, C., Barthelson, R. A., Lambert, G. M., and Galbraith, D. W. (2008). Global characterization of cell-specific gene expression through fluorescence-activated sorting of nuclei. Plant Physiol. 147, 30-40. doi: 10.1104/pp.107.115246

Zhang, H., Gao, S., Lercher, M. J., Hu, S., and Chen, W.-H. (2012). EvolView, an online tool for visualizing, annotating and managing phylogenetic trees. Nucleic Acids Res. 40, W569-W572. doi: 10.1093/nar/gks576

Conflict of Interest Statement: The authors declare that the research was conducted in the absence of any commercial or financial relationships that could be construed as a potential conflict of interest.

Received: 22 July 2013; accepted: 10 October 2013; published online: 05 November 2013.

Citation: Xu S, Guerra D, Lee U and Vierling E (2013) S-nitrosoglutathione reductases are low-copy number, cysteine-rich proteins in plants that control multiple developmental and defense responses in Arabidopsis. Front. Plant Sci. 4:430. doi: 10.3389/fpls. 2013.00430

This article was submitted to Plant Physiology, a section of the journal Frontiers in Plant Science.

Copyright (C) 2013 Xu, Guerra, Lee and Vierling. This is an open-access article distributed under the terms of the Creative Commons Attribution License (CC BY). The use, distribution or reproduction in other forums is permitted, provided the original author(s) or licensor are credited and that the original publication in this journal is cited, in accordance with accepted academic practice. No use, distribution or reproduction is permitted which does not comply with these terms. 